\title{
Dyons in Supersymmetric Restricted Quantum Chromodynamics(SRCD)
}

\section{J M S RANA}

Department of Physics,HNB Garhwal University, SRT Campus, Badshahithaul(Tehri-Garhwal),India

Email : ranajms@gmail.com

\section{ABSTRACT}

The Restricted Quantum Chromodynamics (RCD) formulated in terms of connections on global spaces has been supersymmetrized, in a general manner, taking only the topological part into considerations. Dyonic supermultiplets have been obtained for the $\mathrm{N}=1$ supersymmetry, quantum mechanically as well as in the supersymmetric version of Georgi-Glashow model for vanishing linear momentum and in the Clifford vacuum. Incorporating the Dyonic color charge and color spin induced as a result of fermion fractionization in Georgi- Glashow model(in presence of an isovector fermion field)into the Supersymmetric Restricted Quantum Chromodynamics in N=1 SUSY limit ,the Lagrangian density has been constructed and SUSY Dyonic solutions have been obtained . Furthermore, the classical mass of the Dyon has been calculated by minimizing the background potential of theory. The eigen value equations of bosonic and fermionic fluctuations have been obtained in the dyonic background gauge and the corresponding one-loop corrections to the Dyonic mass are calculated and it has been shown that one-loop quantum corrections lead no change in classical mass of Dyon.

8th Conference Quark Confinement and the Hadron Spectrum

Mainz, Germany

1-6 September, 2008 


\section{Introduction}

The non-trivial topological structure and corresponding classical solutions of non-Abelian gauge theories, enforces us to speculate the existence of built-in-duality, which may play an important role in understanding some non-perturbative aspects of QCD e.g. chiral symmetry breaking and quark confinements etc. It is conjectured that the non-Abelian gauge theories may express an exact electro-magnetic duality that exchanges solitons with elementary quanta and weak coupling with strong coupling. QCD has been formulated as a dual gauge theory called Restricted quantum chromodynamics (RCD) which exhibits built-in-dual structure and its dual dynamics guarantees the confinement of colored fluxes associated with dyonic quarks through the mechanism of generalized Meissner effect. Constructing the Lagrangian density in the $\mathrm{N}=1$ supersymmetric $\mathrm{RCD}$ (topological part only) in terms of the isotriplet gauge field and its fermionic superpartner, supersymmetric dyonic solutions are obtained and the classical mass of the dyon is calculated by minimizing the background potential of the theory.

\subsection{Restricted quantum chromodynamics $(\mathrm{RCD})$ - A brief review:}

The mathematical foundation of RCD lies in the observation that the non-Abelian gauge symmetry does allow an extra internal symmetry, called magnetic symmetry, imposed by insisting on the following gauge covariant condition;

$$
D_{\mu} \hat{m}=\partial_{\mu} \hat{m}+q^{*} V_{\mu} \times \hat{m}=0
$$

Where, $\hat{m}$ is an arbitrary multiplet and constitute an adjoint representation of group $\mathrm{G}$ whose little group is assumed to be Cartan's subgroup at each space-time point. Normalising $\hat{m}$ (i.e. $\hat{m}^{2}=1$ ) and choosing $\mathrm{G}=\mathrm{SU}(2)$, the gauge covariant condition in eq. (1) gives the following form of generalized restricted gauge potential;

$$
V_{\mu}=-i V_{\mu}^{*} \hat{m}-\frac{1}{q^{*}} \hat{m} \times \partial_{\mu} \hat{m}
$$

Such that $\hat{m} \cdot V_{\mu}=-V_{\mu}^{*}$ is the unrestricted Abelian component while the remaining part of $V_{\mu}$ is completely determined by magnetic symmetric requirement. The generalized restricted gauge field strength corresponding to $V_{\mu}$ can be constructed in the following form;

$$
G_{\mu v}=\varsigma_{\mu v}+q^{*}\left[V_{\mu} \times V_{v}\right]=\left(-i F_{\mu \nu}+H_{\mu \nu}\right) \hat{m}
$$

Identification of $F_{\mu \nu}$ and $H_{\mu \nu}$ as generalized electric and magnetic field strengths,obviously manifest striking duality between electric and magnetic fields which can be made more explicit in magnetic gauge by imposing a gauge transformation $\mathrm{U}$, such that:

$$
\hat{m} \quad \underline{U} \quad \hat{\xi}_{3}=(0,0,1)^{T} .
$$

The gauge invariant SU (2), RCD lagrangian may be written in the following form;

$$
l_{R}=-\frac{1}{4} G_{\alpha \mu \nu} G^{\alpha \mu \nu}+i \bar{\psi}^{\alpha} \gamma^{\mu} D_{\mu} \psi_{\alpha}-m \bar{\psi}^{\alpha} \psi_{\alpha}
$$


Describing the dyonic source by a complex field operator $\phi$, RCD lagrangian (eq. (7)) is modified to : $\quad L_{R}=l_{R}+l_{C}$

Where, $1_{\mathrm{C}}$ is obtained as $l_{C}=\left|\left(\partial_{\mu}+i \frac{4 \pi}{|q|} V_{\mu}^{\prime}\right)\right|^{2}-V\left(\phi^{*} \phi\right)$

$V\left(\phi^{*} \phi\right)$ is the effective potential. we obtain the following phenomenological Lagrangian, in the magnetic gauge, just as Ginzburg-Landau Lagrangian of superconductivity:

$$
\begin{aligned}
L_{R} & =-\frac{1}{4} F_{\mu \nu} F^{\mu v}-\frac{1}{4} H_{\mu \nu} H^{\mu v}-\frac{i}{2}\left(H_{\mu \nu} F^{* \mu v}-F_{\mu \nu} H^{* \mu v}\right) \\
& +\bar{\varphi}_{+} i \gamma^{\mu}\left\{\partial_{\mu}+\frac{q^{*}}{2}\left(-i V_{\mu}^{*}+W_{\mu}\right)\right\} \varphi_{+}+\bar{\varphi}_{-} i \gamma^{\mu}\left\{\partial_{\mu}-\frac{q^{*}}{2}\left(-i V_{\mu}^{*}+W_{\mu}\right)\right\} \varphi_{-}+m\left(\bar{\varphi}_{+} \varphi_{+}+\bar{\varphi}_{-} \varphi_{-}\right) \\
& +\left|\left\{\partial_{\mu}+i \frac{4 \pi}{|q|}\left(-i V_{\mu}^{*}+W_{\mu}\right)\right\} \phi\right|^{2}-V\left(\phi^{*} \phi\right)
\end{aligned}
$$

\subsubsection{Supersymmetric Dyons and Restricted Gauge Theory:}

Dyons appear in RCD only through restricted part of generalized potential,so the gauge potential and field strength now may be written in the following modified form:

$V_{\mu}=-\frac{1}{|q|} \hat{m} \times \partial_{\mu} \hat{m}, \quad$ and $\quad G_{\mu \nu}^{a}=\frac{1}{|q|}\left(2 \varepsilon^{a b c} \partial_{\mu} m_{b} \partial_{\nu} m_{c}+m^{a} \varepsilon^{b c d} m_{b} \partial_{\mu} m_{c} \partial_{\nu} m_{d}\right)$

The supersymmetric RCD obtained by modifying the Lagrangian density into the form:

$L=-\frac{1}{4} G_{\mu \nu}^{a} G_{a}^{\mu v}+\frac{1}{2} \bar{\lambda}^{a} \gamma^{\mu} D_{\mu} \lambda_{a}+\frac{1}{2} D_{\mu} m^{a} D^{\mu} m_{a}+\frac{1}{2}|q| \varepsilon_{a b c} m^{a} \bar{\lambda}^{b} \gamma_{5} \lambda^{c}-V\left(m m^{*}\right)$,

where $\lambda^{a}$ constitutes the isotriplet of fermionic field, $\gamma^{\mu}$ are Dirac matrices, $\gamma_{5}=\gamma_{1} \gamma_{2} \gamma_{3} \gamma_{4}$,

The background potential $V\left(\mathrm{~mm}^{*}\right)$ has been constructed in the form

$$
V\left(m m^{*}\right)=|q|^{2}\left[\left(m^{a} m_{a}^{*}\right)^{2}-\left(m^{a} m_{a}^{*}\right)\left(m^{b} m_{b}^{*}\right)\right]
$$

SUSY Lagrangian of topological part of RCD is then given in the following form,

$$
\begin{aligned}
& L=-\frac{1}{|q|^{2}}\left[\Gamma_{\mu v b c}\left(\Gamma^{\mu v b c}-\Gamma^{\mu v c b}\right)+\frac{1}{2} \varepsilon_{a j k} \varepsilon^{b c d} m^{a} m_{b} \Gamma_{\mu v c d} \Gamma^{\mu v j k}\right. \\
& \left.+\frac{1}{2} \varepsilon^{a b c} \varepsilon_{j k l} m_{a} m^{j} \Gamma_{\mu v b c} \Gamma^{\mu \nu k l}+\frac{1}{2} \varepsilon^{b c d} \varepsilon_{j k l} m_{b} m^{j} \Gamma_{\mu v c d} \Gamma^{\mu v k l}\right] \\
& +\frac{1}{2} \bar{\lambda}^{a} \gamma^{\mu}\left[\partial_{\mu} \lambda_{a}+\left(m_{a} \partial_{\mu} m_{b} \lambda^{b}-m_{b} \partial_{\mu} m_{a} \lambda^{b}\right)\right] \\
& +\frac{1}{2}\left[\partial_{\mu} m^{a}+\left(m^{a} \partial_{\mu} m^{b} m_{b}-m^{b} \partial_{\mu} m^{a} m_{b}\right)\right]^{2} \\
& +\frac{1}{2}|q| \varepsilon_{a b c} m^{a} \bar{\lambda}^{b} \gamma_{5} \lambda^{c}-V\left(m m^{*}\right),
\end{aligned}
$$

In order to check the supersymmetric invariance of this Lagrangian, we apply the following supersymmetric transformations:

$$
\delta m^{a}=\bar{\alpha} \gamma_{5} \lambda^{a}
$$




$$
\begin{aligned}
& \delta V_{\mu}^{a}=-\frac{1}{|q|} \varepsilon^{a b c} \bar{\alpha}\left(\gamma_{5} \lambda_{b} \partial_{\mu} m_{c}+m_{b} \gamma_{5} \partial_{\mu} \lambda_{c}\right), \\
& \delta \lambda^{a}=\frac{1}{|q|} \alpha \sigma^{\mu v}\left(2 \varepsilon^{a b c} \Gamma_{\mu v b c}+m^{a} m_{b} \varepsilon^{b c d} \Gamma_{\mu v c d}\right)-i \alpha \gamma_{5} \gamma_{\mu} m^{a} m_{b} \gamma^{\mu} m^{b} .
\end{aligned}
$$

Under these transformations, $\quad \delta L=0$
Provided we assume the supersymmetric conditions,

$$
\lambda_{5}\left(\bar{\lambda} \times \partial_{\mu} \hat{m}+\hat{m} \times \partial_{\mu} \bar{\lambda}\right)=-i|q| \gamma_{\mu} \bar{\lambda}, \quad\left[m_{b}, \bar{\alpha}\right]=0, \quad\left[m_{b}, \gamma_{5}\right]=0
$$

which give the generalization of the Majorana condition and the Weyl condition.

From the Lagrangian density we get the following form of energy-momentum density tensor,

$$
\begin{aligned}
T^{\mu \nu}= & D^{\mu} m_{a} D^{v} m^{a}+\frac{1}{2} G_{a}^{\mu \lambda} G_{\lambda}^{a v}-g^{\mu v}\left[-\frac{1}{4} G_{i j}^{a} G_{a}^{i j}+\frac{1}{2} \bar{\lambda}^{a} \gamma^{i} D_{i} \lambda_{a}\right. \\
& \left.+\frac{1}{2} D_{i} m^{a} D^{i} m_{a}+\frac{1}{2}|q| \varepsilon_{a b c} m^{a} \bar{\lambda}^{b} \gamma_{5} \lambda^{c}-V\left(m m^{*}\right)\right] .
\end{aligned}
$$

Setting $\mu=v=0$ in this relation, integrating $T^{\infty}$ over three-space, the classical mass of the dyon comes out to be $\quad M=\frac{v}{\sqrt{2}}|q|=M_{\text {classical }}$

In the dyonic background fields $V_{\mu D}^{a}=V_{\mu}^{a}-\delta V_{\mu}^{a}, \quad m_{D}^{a}=m^{a}-\delta m^{a}$,

the bosonic part of the Lagrangian density (14) may be written as

$$
\begin{aligned}
L_{B}= & L_{D}-\frac{1}{2}\left|\left(D_{v} \delta V_{\mu}^{a}\right)\right|^{2}+\frac{1}{2}\left(D_{\mu} \delta V_{v}\right)\left(D^{v} \delta V^{\mu}\right)^{*}-\frac{1}{4}|q|^{2}\left|\left(\delta V_{\mu b} \delta V_{v c}\right)\right|^{2} \\
& -\frac{1}{2} \operatorname{Re}\left[|q| \varepsilon_{a b c} G_{\mu \nu D}^{a} V^{* \mu b} \delta V^{* v c}\right]-\frac{1}{2}\left(D_{\mu} \delta m^{a}\right)\left(D^{\mu} \delta m_{a}\right)^{*}-\frac{1}{2}|q| \varepsilon_{a b c}\left(D_{\mu} m^{a}\right) V^{* \mu b} m^{c} \\
& +\frac{1}{2}|q| \varepsilon_{a b c}\left(D_{\mu} \delta m^{a}\right) \delta V^{* \mu b} \delta m^{c}+\frac{1}{2}|q| \varepsilon^{a b c}\left(D^{\mu} m_{a}\right)^{*} \delta V_{\mu b} m_{c}+\frac{1}{2}|q|^{2}\left[\delta V_{\mu b} m_{c}\right. \\
& +\frac{1}{2}|q|^{2}\left[\left(\delta V_{\mu b} m^{a}\right)^{2}-\left(\delta V_{\mu b}\right)^{2}\left(m_{a}\right)^{2}\right],
\end{aligned}
$$

where $L_{D}$ is the dyonic background Lagrangian given by

$$
L_{D}=-\frac{1}{4} G_{\mu \nu D}^{a} G_{a D}^{* \mu \nu}+\frac{1}{2}\left(D_{\mu} m_{D}^{a}\right)\left(D^{\mu} m_{D a}\right)-V\left(m_{D}^{* a} m_{D a}\right)
$$

To this Lagrangian we add: (i) Gauge-fixing term: the gauge-fixing term is given by $L_{g f}=-\frac{1}{2} f^{a} f_{a}=-\frac{1}{2}\left(D_{\mu} \delta V^{\mu}\right)^{2}+|q| \varepsilon^{a b c}\left(D_{\mu} \delta V^{\mu}\right)_{a} m_{b} \delta m_{c}+|q|^{2}\left(m^{a} m_{b}-m^{2} \delta_{b}^{a}\right) \delta m_{a} \delta m^{b}$.

(ii) Faddeev-Popov ghost term: This term is given by $L_{F P}=-c_{a}^{*}\left(\delta f^{a} / \delta \theta^{b}\right) c_{b}$,

$$
L_{F P}=c_{a}^{*}\left[\frac{1}{|q|}\left[D^{\mu}\left(m^{a} \partial_{\mu} m^{b}+m_{b} \partial_{\mu} m^{a}\right)\right]+|q|^{2}\left(m^{a} m^{b}-m^{2} \delta^{a b}\right)\right] c_{b}
$$

The total Lagrangian for Bosonic part be then written as: $L=L_{B}+L_{g f}+L_{F P}$.

One-loop contributions to dyonic mass: $\Delta M_{\text {Bose }}=\Delta M_{B}=\sum \omega_{B}+\frac{1}{2} \sum \omega_{B_{0}}-\sum \omega_{G}$,

i.e may be written as , $\quad M_{\text {Bose }}=\frac{1}{2} \sum \omega_{B}$ 
Similarly fermionic fluctuation to dyonic mass: $\Delta M_{F}=M_{F e r m i}=-\frac{1}{2} \sum \omega_{F}$.

Total one-loop quantum correction to dyon mass $\Delta M=\Delta M_{B}+\Delta M_{F}=\frac{1}{2}\left[\sum \omega_{B}-\sum \omega_{F}\right]$.

Dyonic mass up to one-loop quantum correction $M=M_{\text {classical }}+\frac{1}{2}\left[\sum \omega_{B}-\sum \omega_{F}\right]$,

But, $\sum \omega_{F}=\sum \omega_{B}$. hence in the supersymmetric limit, the non-Abelian theory of dyons in RCD falls apart, in the correct way, into degenerate supermultiplets.

\subsubsection{Results and Discussions}

The Lagrangian density is supersymmetric for the generalized Majorana and Weyl conditions leading to supersymmetric dyonic solutions with classical mass as predicted by Julia and Zee. In the dyonic background field the bosonic part of the supersymmetric Lagrangian gives bosonic fluctuations and fermionic part in turn yields the fermionic fluctuation leading to one-loop corrections to dyonic mass which shows no change in classical limit. This SUSY dyonic model and the vanishing of the quantum corrections may be used for proving Montonen and Olive conjecture. All these results of the supersymmetrized RCD agree with conclusions drawn by D'Adda et al using dimensionally reduced supersymmetrized pure Yang-Mills theory in six dimensions.

\section{References}

[1] D.I. Olive : Exact electromagnetic duality, hep-th/9612192.

[2] N. Sieberg and E. Witten : Nucl. Phys. B 426 (1994) 19 ; B 430 (E) (1994) 485

[3] G. ' t Hooft : Nucl. Phys. B 79 (1976) 276.A. Polyakov : JETP Lett. 20 (1974) 194.

[4] C. Montonen and D. Olive : Phys. Lett. B72 (1977)117.

[5] J.M.S. Rana, H.C. Chandola and B.S. Rajput : Prog.Theor. Phys. 82(1989)153 ; Ind. J. Pu \& Appl. Phys. 26 (1988)587.H.C. Chandola, B.S. Rajput, J.M.S. Rana and S. Sah: IL Nuov. CIM. A106(1993) 509.

[6] Y.M. Cho: Phys. Rev. D 23 (1981) 2415; Phys. Rev. Lett. 46 (1981)302. J.M.S. Rana , H.C. Chandola and B.S. Rajput : Can. J. Phys. 69 1993(1441.

[7] J.M.S. Rana, H.C. Chandola, and B.S. Rajput, Ind. J. Pure Appl. Phys. 26,587(1988) ; Prog. Theor. Phys. 82, 153(1989).

[8] Y.M. Cho, Phys. Rev. D 21, 1080 (1980); Report No. MZ.TH/82-05, Uni of Mainz,1982.

[9] B. Julia and A. Zee, Phys. Rev. D11, 2227(1975).

[10] C. Montonen and D. Olive, Phys. Lett. 72B, 177(1977).

[11] A. D,Adda, R. Horsley, and P. Di Vecchia, Phys. Lett. 76B, 298 (1978). 